\title{
Activation of ERK/CREB/BDNF pathway involved in abnormal behavior of neonatally Borna virus- infected rats
}

This article was published in the following Dove Press journal:

Neuropsychiatric Disease and Treatment

\author{
Chenmeng $\mathrm{Li}^{1-3, *}$ \\ Xiaoyan $\mathrm{Xu}^{2-4, *}$ \\ Xiong Zhang ${ }^{2-4, *}$ \\ Ke Cheng ${ }^{2,3, *}$ \\ Yujie Guo ${ }^{1-3}$ \\ Jie Jie ${ }^{1-3}$ \\ Hua Guo ${ }^{1-3}$ \\ Yong $\mathrm{He}^{1-3}$ \\ Chanjuan Zhou ${ }^{2,3}$ \\ Siwen Gui ${ }^{2,3}$ \\ Xiaogang Zhong ${ }^{2,3}$ \\ Haiyang Wang ${ }^{2-4}$ \\ Peng Xie ${ }^{1-4}$
}

'Department of Neurology, Yongchuan

Hospital of Chongqing Medical

University, Chongqing 402460,

China; ${ }^{2}$ Department of Neurology Institute of Neuroscience and

Collaborative Innovation Center for

Brain Science, Chongqing Medical

University, Chongqing 400016,

China; ${ }^{3}$ Chongqing Key Laboratory

of Neurobiology, Chongqing 400016 ,

China; ${ }^{4}$ Department of Neurology,

The First Affiliated Hospital of

Chongqing Medical University,

Chongqing 400016, China

*These authors contributed equally to this work

Correspondence: Peng Xie Department of Neurology, The First Affiliated Hospital of Chongqing Medical University, No I Yixue Road, Yuzhong

District, Chongqing 400016, China

Tel +862368485490

Email xiepeng@cqmu.edu.cn
Background: Neuropsychiatric disorders are devastating illnesses worldwide; however, the potential involvement of viruses in the pathophysiological mechanisms of psychiatric diseases have not been clearly elucidated. Borna disease virus (BDV) is a neurotropic, noncytopathic RNA virus.

Materials and methods: In this study, we infected neonatal rats intracranially with BDV Hu-H1 and Strain V within 24 hours of birth. Psychological phenotypes were assessed using sucrose preference test, open field test, elevated plus maze test, and forced swim test. The protein expression of ERK/CREB/BDNF pathway was assessed by Western blotting of in vitro and in vivo samples.

Results: Hu-H1-infected rats showed anxiety-like behavior 8 weeks postinfection while Strain V-infected rats demonstrated a certain abnormal behavior. Phosphorylated ERK1/2 was significantly upregulated in the hippocampi of Strain V-and Hu-H1-infected rats compared with control rats, indicating that Raf/MEK/ERK signaling was activated.

Conclusion: The data suggested that infection of neonatal rats with BDV Hu-H1 and Strain V caused behavioral abnormalities that shared common molecular pathways, providing preliminary evidences to investigate the underlying mechanisms of psychiatric disorders caused by BDV.

Keywords: Borna disease virus, ERK/CREB/BDNF signaling pathway, neonatal Borna disease

\section{Introduction}

Neuropsychiatric disorders such as major depression, anxiety, and schizophrenia are devastating illnesses worldwide. The etiology of neuropsychiatric diseases has been connected with disturbances of neurogenesis and neurotransmitters, dysfunction of the hypothalamic-pituitary-adrenocortical axis, effects of environmental toxicants, genetic factors, and many other factors. Borna disease virus (BDV) is a neurotropic, noncytopathic RNA virus belonging to the family Bornaviridae in the order Mononegavirales. BDV persistently infects the central nervous system of a wide range of vertebrate species, ${ }^{1,2}$ causing neurological diseases and behavioral disorders. Over the past several decades, substantial evidence has indicated that BDV may be involved in human psychiatric disorders. ${ }^{3-9}$ It has been suggested that BDV may play a complex role in the etiology of such disorders, influencing T-cell-mediated cytotoxicity, microglial cytokine-mediated neurotoxicity, and disturbances in the dopaminergic, GABAergic and glutamatergic systems. ${ }^{10-15}$ Nevertheless, the detailed neuropathogenesis of BDV remains largely unknown. Thus, the aim of this study was to illustrate the potential relationships between BDV infection and neuropsychiatric disorders.

Strain V is a horse-derived, rabbit-adapted, laboratory reference BDV strain. BDV Hu-H1 is a human strain of BDV that was isolated from the peripheral blood 
mononuclear cells of patients with major mood disorders by German scientists in $1996 .{ }^{16}$ In our previous work, we showed that $\mathrm{Hu}-\mathrm{H} 1$ but not Strain $\mathrm{V}$ inhibited proliferation and promoted apoptosis of human oligodendroglial (OL) cells. ${ }^{17}$ BDV Hu-H1 and Strain V shared a high degree of genetic homology but exerted different biological effects (our unpublished data). Thus, we deemed that unique changes may have been adapted by these two BDV strains, which have undergone continuous evolution, ${ }^{18}$ to their surroundings, and we hypothesized that they would cause different behaviors in rats.

The Raf/MEK/ERK signaling cascade belongs to the mitogen-activated protein kinase (MAPK) signaling pathway family and is directly relevant to a variety of processes including neuronal cell proliferation, differentiation, growth, and apoptosis as well as in the activation of many RNA viruses. ${ }^{19,20}$ A previous study demonstrated that the pathogenesis of schizophrenia was associated with abnormalities of ERK signaling in the thalamus and cerebellum. ${ }^{21}$ Moreover, it was also shown that acute blockade of MEK/ERK signaling in mice conferred a depressive-like phenotype and that the resulting alleviative behavioral actions stopped after oral antidepressants were administered. ${ }^{22}$ A similar report indicated that blocking ERK phosphorylation could cause autistic behavior in mice. ${ }^{23}$ ERK1/2 signaling was one of the key molecular pathways involved in the etiology of autism. ${ }^{24}$ In addition, the ERK/CREB/BDNF pathway also participated in the pathophysiology of major depressive disorder. ${ }^{25}$ Based on this, in this study we aimed to investigate the relationship between BDV and ERK/CREB/BDNF pathway.

In our previous study, BDV Hu-H1 was used to infect human OL cells and resulted in significant disturbance of the Raf/MEK/ERK signaling pathway. ${ }^{26}$ The results demonstrated that the protein expression of p-ERK1/2 and p-RSK were significantly upregulated, and p-MSK was notably downregulated in BDV Hu-H1-infected OL cells compared with noninfected OL cells. Consistent with this, BDV CRP4 caused constitutive activation of ERK1/2 signaling in PC12 cells, impairing neuronal differentiation. ${ }^{27}$ All of the aforementioned studies were performed in vitro and were not pursued in vivo. In this study, using intracerebroventricular injection, we established a postnatal rat model of BDV infection and used it to investigate how two different BDV strains (Hu-H1 and Strain V) induced behavioral changes. We were particularly interested in the effects of BDV infection on the ERK/CREB/BDNF pathway both in vitro and in vivo, and the relevance of this model for further investigations of psychiatric diseases.

\section{Materials and methods Animals and ethics statement}

Pregnant Sprague-Dawley (SD) rats $(n=3)$ at $16-18$ days of gestation were provided by the animal center of Chongqing Medical University (Chongqing, China) and kept in individual cages before the newborn rats were separated from the mother. Mothers and their litters $(n=36$ and $n=12$ per group) were kept in rectangular polypropylene cages with wood chip bedding and covered with metal grids bearing grooves supporting the food and water bottles. The BDV was extracted from squamous epithelial cells via freezing and thawing cycles in sterile PBS buffer. Each neonatal rat (within 24 hours of birth) received an injection of $30 \mu \mathrm{L}$ $\left(1 \times 10^{4} \mathrm{FFU} / \mathrm{mL}\right)$ of BDV inoculum (both strains were donated by Professor Hanns Ludwig, Free University of Berlin, Germany) or an equal volume of PBS (HyClone, Thermo Fisher Scientific, Waltham, MA, USA) into the left lateral ventricle using a $50-\mu \mathrm{L}$ Hamilton syringe as previously described. ${ }^{28}$ Throughout the experiment, rats were maintained on a 12-hour light-dark cycle under a constant temperature $\left(21^{\circ} \mathrm{C} \pm 1^{\circ} \mathrm{C}\right)$. After postnatal day 21 , the growing rats were separated and housed in individual cages where they were given food and water ad libitum. The experimental procedures followed the National Institutes of Health Guide for the Care and Use of Laboratory Animals and were approved by the Ethics Committee of Chongqing Medical University.

\section{Experimental procedures}

Eight weeks postinfection, sucrose preference test (SPT), open field test (OFT), elevated plus maze (EPM) test, and forced swim test (FST) were successively administered under the same conditions. Each behavioral test was administered in this order: 1) control group, 2) BDV Hu-H1 group, and 3) BDV Strain V group. Experiments were conducted from 9:00 am to 4:00 pm. Illumination was provided using a $40 \mathrm{~W}$ bulb in a soundproof room.

\section{SPT}

Rodents are very sensitive to sweet solution and this is an effective reward for rats. The degree of anhedonia in rats is usually assessed by the amount of sugar in water and their sucrose preference (SP). Most scholars believe that a decrease in SP is a major characteristic of the depressive rat. The SPT was conducted based on the procedure outlined by Cheng et al. ${ }^{29}$ Rats were trained to drink $1 \%(\mathrm{w} / \mathrm{v})$ sucrose solution for 3 days before the test. After 12 hours of water and food deprivation, two identical bottles (containing 1\% 
sucrose solution or water) were weighed before testing. The two bottles were reweighed 2 hours later. The SP was calculated as: $\mathrm{SP}(\%)=($ sucrose solution intake/[sucrose solution intake + water intake] $) \times 100$.

\section{OFT}

The OFT is a method to evaluate the locomotor activity, exploration behavior, and tension of experimental animals in novel surroundings. Each rat was allowed to freely explore the square black apparatus for 5 minutes. Testing was recorded using an automated video tracking system (SMART, Panlab, Barcelona, Spain). The apparatus was wiped with $70 \%(\mathrm{v} / \mathrm{v})$ ethanol to remove odors between each test. The detailed process was described previously. ${ }^{29}$ The distance traveled, number of entries into the center zone, and rearing behavior (number of events) were calculated.

\section{EPM test}

The EPM test is used to evaluate the anxiety of experimental animals based on rodents' aversion for open spaces. Less time spent in the closed arm and longer time in the open arm indicated lower level of anxiety. Locomotor activity was also assessed by measuring the total traveled distance. The EPM was conducted following a published procedure. ${ }^{30}$ Briefly, rats were placed in the center of the EPM apparatus facing a closed arm. Every activity within 5 minutes were recorded using an automated video tracking system. The total distance traveled, number of entries, and time spent in the open arms were measured.

\section{FST}

The FST is regarded as a reliable method for assessing depression in experimental animals. It was performed after the EPM was completed..$^{30}$ The first swimming pretest was conducted for 15 minutes in a glass cylinder containing water at a suitable temperature $\left(23^{\circ} \mathrm{C} \pm 2^{\circ} \mathrm{C}\right)$. The animals were then gently dried using a heating radiator and put back in their cages. Twenty-four hours later, the above experiment was conducted for 5 minutes. All processes were recorded using an automated video tracking system. The immobility time was counted.

\section{Sacrifice and tissue harvesting}

After the completion of behavioral tests, rats were anesthetized using 10\% chloral hydrate (BBI Life Sciences Corporation, Shanghai, China) overdose and quickly perfused through the left ventricle with precooled sodium chloride.
The hippocampus was isolated from brain and frozen at $-80^{\circ} \mathrm{C}$ for further experiments.

\section{Cell culture and viral infection}

Pregnant SD rats at 16-18 days gestation were anesthetized as described above. Fetuses were quickly isolated from the uterus while the maternal rat was under deep anesthesia. Fetal rats were transferred onto culture dishes placed on ice packs disinfected with $75 \%$ ethanol. The embryos were quickly decapitated, and their brains were immediately submerged in ice-cold DMEM (HyClone). The hippocampus was then isolated. After carefully removing the meninges, the hippocampus was cut into small pieces using a special knife and incubated with preheated $0.125 \%$ trypsin-EDTA ( $0.25 \%$ trypsin; Thermo Fisher Scientific) diluted in preheated PBS. The cell suspensions were filtered using a $75-\mu \mathrm{m}$ nylon strainer. The dissociated cells were seeded at a density of $1.2 \times 10^{6}$ cells/well on 6 -well plates precoated with poly-L-lysine (Sigma-Aldrich Co., St Louis, MO, USA). After 5-6 hours, the culture medium was replaced with neurobasal medium. Thereafter, the two BDV strains were added $(\mathrm{MOI}=1)$, and 3 hours later the culture medium was exchanged. All formula reagents and protocols were described previously. ${ }^{31}$

\section{Immunofluorescence}

We performed immunofluorescence assay at day 9 postinfection according to previous research on primary hippocampal cells. ${ }^{32}$ Cells were fixed in $4 \%(\mathrm{w} / \mathrm{v})$ paraformaldehyde for 15 minutes, then treated with $0.25 \%(\mathrm{v} / \mathrm{v})$ Triton X-100 for 10 minutes, and blocked with $5 \%(\mathrm{w} / \mathrm{v})$ BSA for 40 minutes. Neurons were incubated with rabbit antiMAP2 antibody (1:2,000 dilution; Beyotime Biotechnology, Shanghai, China) and mouse anti-P24 monoclonal antibody (1:200 dilution) overnight at $4^{\circ} \mathrm{C}$, followed by Cy3-labeled goat anti-rabbit IgG antibody (1:500 dilution; Beyotime Biotechnology) and FITC-labeled goat anti-mouse IgG (1:500 dilution; Beyotime Biotechnology) for 1 hour. Finally, the cells were stained with DAPI (1:5,000 dilution; Beyotime Biotechnology). The cells were imaged using an inverted fluorescence microscope (Nikon, Tokyo, Japan).

\section{Western blotting}

For the identification of proteins involved in the ERK/BDNF/ CREB pathway, hippocampi were lysed in RIPA buffer (Beyotime Biotechnology). Cultured primary hippocampal neurons were lysed using a protease inhibitor cocktail (KeyGEN Biotech Co., Ltd., Jiangsu, China). Protein concentration 
was determined using bicinchoninic acid assay (Beyotime Biotechnology). Proteins were separated using 10\% SDSPAGE and transferred to polyvinylidene difluoride membranes (EMD Millipore, Billerica, MA, USA). The membranes were blocked with 5\% (w/v) nonfat dried milk (BBI Life Sciences Corporation) for 3-4 hours, then incubated with the following antibodies overnight at $4^{\circ} \mathrm{C}$ : phospho-c-Raf (Ser338) rabbit monoclonal antibody (Cell Signaling Technology, Danvers, MA, USA; 1:1,000); phospho-MEK1/2 (Ser217/221) rabbit monoclonal antibody (Cell Signaling Technology; 1:1,000); phospho-ERK1/2 (Thr202/Tyr204) rabbit monoclonal antibody (Cell Signaling Technology; 1:2,000); phosphop90RSK (Ser380) rabbit monoclonal antibody (Cell Signaling Technology; 1:1,000); phospho-MSK (Thr581) rabbit monoclonal antibody (Cell Signaling Technology; 1:1,000); BDNF rabbit polyclonal antibody (Beyotime Biotechnology; 1:500); anti-CREB-1 and anti-phospho-CREB-1 (Bioss Antibodies, Woburn, MA, USA; 1:1,000); and P40 or P24 antibodies (Abgent, Inc., Santa Diego, CA, USA; 1:5,000). Thereafter, the membranes were treated with horseradish peroxidase-conjugated secondary antibodies for 2 hours. Blots were visualized using an enhanced chemiluminescence kit (EMD Millipore), and each sample was quantified using Quantity One software (Bio-Rad Laboratories Inc., Hercules, CA, USA). All experiments were performed in triplicate under the same conditions.

\section{Statistical analyses}

All data were presented as mean \pm standard error of the mean (SEM) and analyzed using SPSS 21.0 (IBM Corporation, Armonk, NY, USA). Data were compared using one-way ANOVA with Tukey's test for post hoc comparisons. Statistical significance was assumed at $P<0.05$.

\section{Results}

$\mathrm{Hu}-\mathrm{H} 1$-infected rats showed an anxiety-like phenotype and Strain V-infected rats showed a certain abnormal behavior.

In the SPT, the percentage of sucrose consumed was $85 \% \pm 2.1 \%$ in PBS-treated rats, $76 \% \pm 3.1 \%$ in $\mathrm{Hu}-$ $\mathrm{H} 1$-infected rats, and $71 \% \pm 5.7 \%$ in Strain V-infected rats. We did not observe any significant differences among these three groups (Figure 1A). There was no anhedonia observed in BDV-infected SD rats, suggesting that no depressive state was induced in these rats in the experimental groups.

In the OFT, a test for general locomotion, first, to evaluate motor function of rats, we tested the average moving speed of all rats. No significance was found among the three groups (Figure 2). This reflects from the possibility that the motor function of rats was not affected by the virus.
Differences in total distance traveled were observed between control rats $(3,438.7 \pm 263.8 \mathrm{~cm})$ and $\mathrm{Hu}-\mathrm{H} 1$-infected rats $(2,235.2 \pm 193.7 \mathrm{~cm}, P<0.01$, Figure 1C), as well as between $\mathrm{Hu}-\mathrm{H} 1$-infected and Strain V-infected rats $(3,065.5 \pm 271.4 \mathrm{~cm}$, $P<0.05$, Figure 1C). Both groups of BDV-infected rats showed significantly diminished vertical exploration and rearing frequency compared with control rats (control: 11.5 \pm 2.2 ; Hu-H1-infected rats: $2.1 \pm 0.5$ and Strain V-infected rats: $2.9 \pm 0.7, P<0.001$, Figure 1D). Hu-H1-infected rats $(0.9 \pm 0.5)$ also made fewer entries into the center zone compared with control rats $(4.5 \pm 0.9, P<0.05$, Figure $1 \mathrm{E})$ and Strain V-infected rats $(7.1 \pm 1.5, P<0.001$, Figure $1 \mathrm{E})$.

In the EPM test, we measured the time spent in the open arm, number of entries, and total distance traveled. The data showed that Strain V-infected rats spent significantly more time (101.7 \pm 22.3 vs $0.7 \pm 0.38$ seconds) and made significantly more entries $(8.8 \pm 1.6$ vs $0.67 \pm 0.36)$ in the open arm of EPM compared with control rats (both $P<0.001$, Figure $1 \mathrm{G}$ and $\mathrm{H})$ and with $\mathrm{Hu}-\mathrm{H} 1$-infected rats $(2.6 \pm 2.0$ seconds, $0.33 \pm 0.19$, both $P<0.001$, Figure $1 \mathrm{G}$ and $\mathrm{H})$. No significant differences were found in these two parameters between $\mathrm{Hu}-\mathrm{H} 1$-infected and control rats $(P>0.5$, Figure $1 \mathrm{G}$ and $\mathrm{H})$. In addition, the total distance traveled significantly decreased for Strain V-infected rats $(1,482.6 \pm 94.6 \mathrm{~cm})$ and Hu-H1infected rats $(1,377.5 \pm 64.3 \mathrm{~cm})$ compared with control rats $(2,191.8 \pm 107.2 \mathrm{~cm}$, both $P<0.001$, Figure 1F). Thus, BDVinfected rats showed significantly decreased locomotion.

In the FST, rats infected with BDV Strain V exhibited decreased immobility time (148.4 \pm 3.3 seconds) compared with control rats (163.3 \pm 2.4 seconds, $P<0.01$, Figure $1 \mathrm{~B})$ and Hu-H1-infected rats (160.6 \pm 4.8 seconds, $P<0.05$, Figure $1 \mathrm{~B})$. Along with the results in the SPT, this finding confirmed that $\mathrm{Hu}-\mathrm{H} 1$-infected rats had no depressive symptoms.

In conclusion, lower locomotor activity, less time spent in the center zone, and diminished vertical exploration in OFT without motor dysfunction, along with lower locomotor activity in the EPM test showed that Hu-H1-infected rats displayed an anxiety-like state at 8 weeks postinfection compared with control rats. Strain V-infected rats showed a weird behavior, in which the rats exhibited decreased mobility in OFT and EPM tests, fear deficiency in the EPM test, and hyperactivity in the FST. The results suggested that Strain V-infected rats had innate anxiety and were unable to recognize potential danger associated with the open arms of the EPM test. Based on the results of existing data, it was difficult to characterize it as an exact phenotype. However, previous study showed that BDV He-80 (BDV strain, also horse-derived, isolated in 1980, Germany) infection of 
A

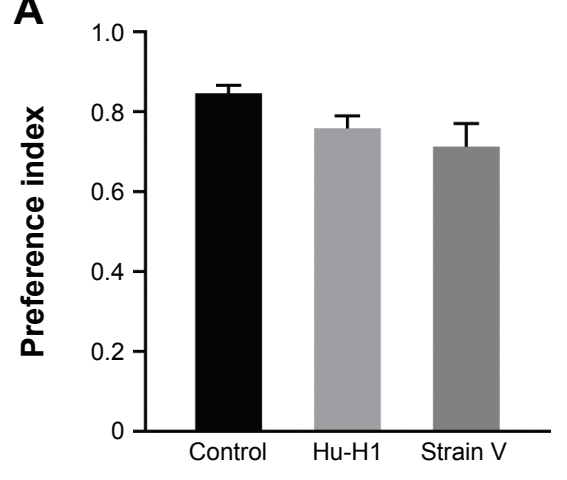

Sucrose preference test

C

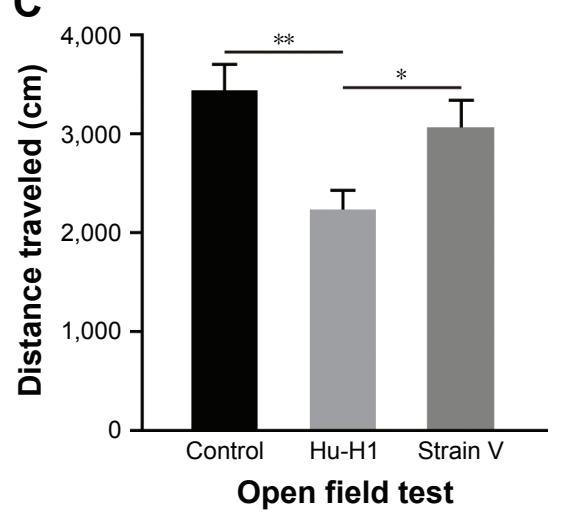

$\mathbf{F}$

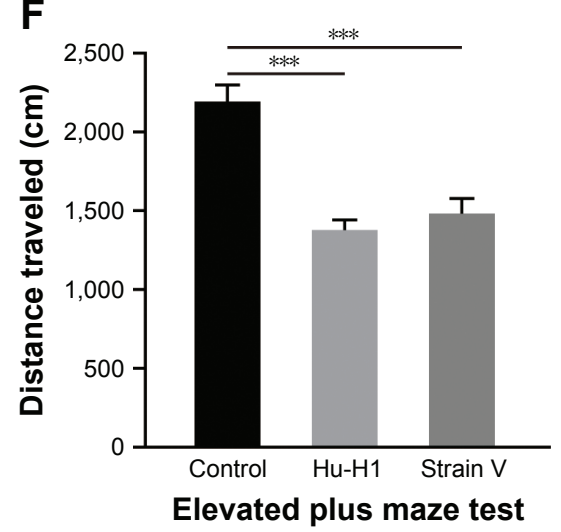

B

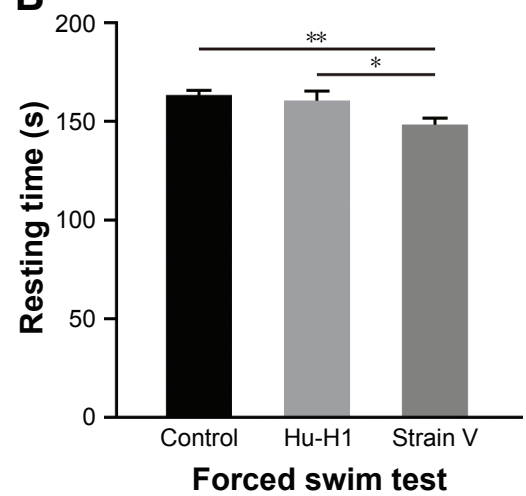

D

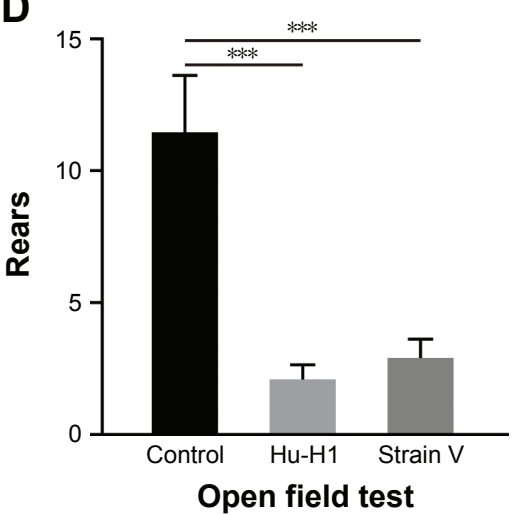

G

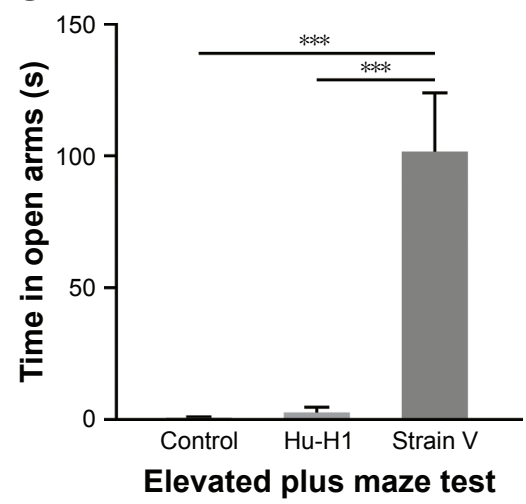

$\mathbf{E}$

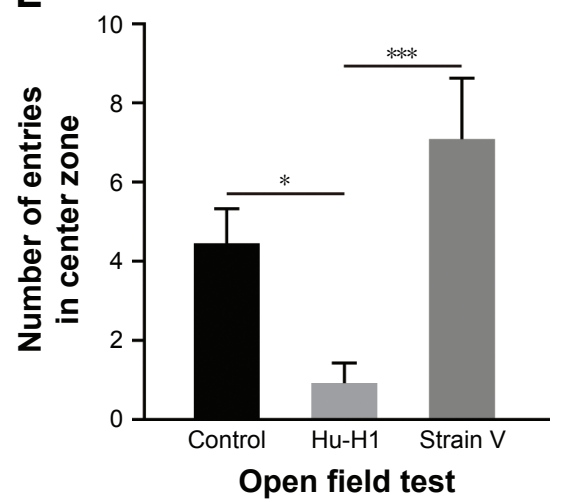

H

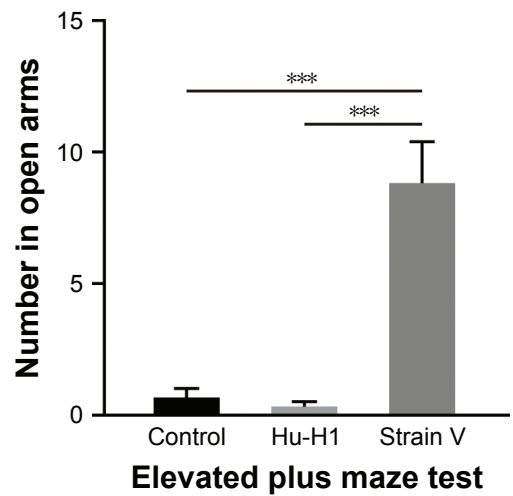

Figure I Animal behavior tests - SPT.

Notes: (A) SP (\%) in the OFT. (B) Immobility time (seconds) in the FST. (C) Total distance covered in the OFT (cm). (E) Number of entries into center zone. (D) Number of rears in the vertical direction in the EPM test. (F) Total distance covered in the EPM test (cm). (G) Time spent in open arms (seconds). (H) Number of entries into open arms in the EPM test. All data are presented as mean \pm SEM ( $n=11-12$ rats/group). Statistical analysis was conducted using ANOVA using post hoc comparisons with Tukey's test. $* P<0.05, * * P<0.01$, and $* * * P<0.001$.

Abbreviations: EPM, elevated plus maze; FST, forced swim test; OFT, open field test; SEM, standard error of the mean; SP, sucrose preference; SPT, sucrose preference test.

neonatal Lewis rats had autism-like behavior. ${ }^{33,34}$ Whether the Strain V-infected rats could be used as an animal model of autism requires further behavioral and pathologic researches. In addition, since horse-derived and human-derived BDV viruses caused very different behaviors in rats, we speculated that they must have different pathogenic mechanisms even though their nucleotide sequences differed at only a few positions (our unpublished data).

\section{High purity and infective efficiency in primary hippocampal neurons}

Representative photomicrographs of cultured primary hippocampal neurons double-labeled with MAP2 and P24 are shown in Figure 3A and B. All nuclei were stained with DAPI (Figure 3C). However, after the infection with BDV, the primary hippocampal cells showed partial apoptosis. When the nuclei of apoptotic cells were stained by DAPI 


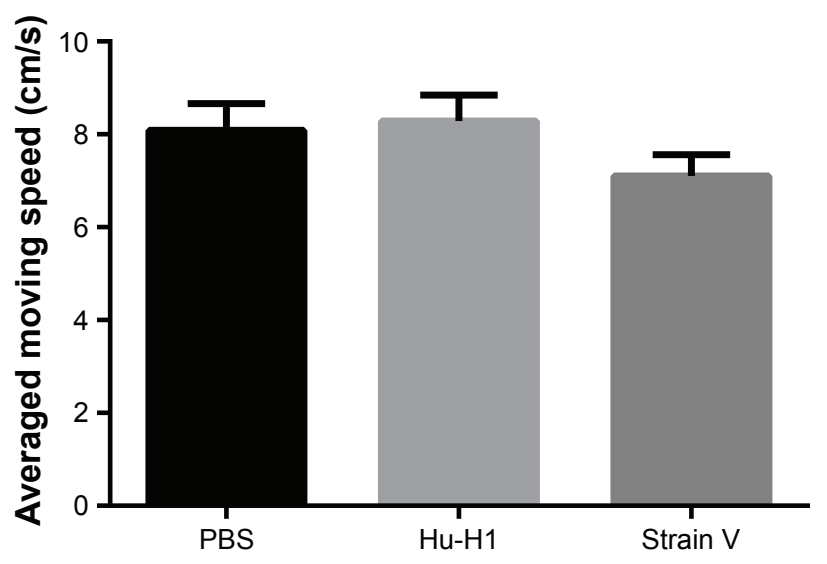

Figure 2 The average moving speed of three groups of rats in OFT. Notes: No significance was found among the three groups. All data are presented as mean \pm SEM. Statistical analysis was performed using ANOVA for post hoc comparisons with Tukey's test.

Abbreviations: OFT, open field test; SEM, standard error of the mean.

(a fluorescent dye that binds strongly to DNA), it presented blue fluorescence under inverted fluorescence microscope without MAP2 staining. Neuronal purity and infection efficiency were evaluated by observing randomly selected cells among three independent experiments in each group. On day 9 , the results demonstrated that the purity of neurons was $>90 \%$ (Figure 3A). The infective efficiency of BDV $\mathrm{P} 24^{+}$neurons was $>85 \%$ (Figure $3 \mathrm{~B}$ ). The merged images are shown in Figure 3D. We carried out the experiment in triplicate under the same conditions.

\section{Activation of ERK/CREB/BDNF signaling pathway in hippocampi of BDV-infected rats}

BDV-specific antibodies (p24 and p40) were used to identify BDV in rats 8 weeks postinfection. The result showed that the rats of the two experimental groups stably expressed the virus in the hippocampus after inoculation with the BDV $\mathrm{Hu}-\mathrm{H} 1$ or Strain V virus compared with the control group (Figure 4). The protein expression levels of p-ERK1/2, p-MEK1/2, p-90RSK, p-MSK1, p-c-Raf, p-CREB, CREB, and BDNF were measured using Western blotting of rat hippocampi. The level of $\mathrm{p}$-ERK1/2 was significantly increased in BDV-infected rat compared with control rats $(P<0.001)$. Both p-90RSK $(P<0.05)$ and p-MSK1 $(P<0.05)$ were found to be significantly upregulated in Hu-H1-infected rats in comparison with control rats, and expression of p-c-Raf was increased in Strain V-infected rats $(P<0.05)$. No significant upregulation was observed in $\mathrm{p}-\mathrm{MEK} 1 / 2$, $\mathrm{p}$-CREB, CREB, and BDNF (Figure 5).

\section{Alteration of ERK/CREB/BDNF signaling pathway in BDV-treated primary hippocampal neurons}

The expression levels of p-ERK1/2, p-MEK1/2, p-90RSK, p-MSK1, p-c-Raf, p-CREB, and BDNF were assessed using Western blotting of primary rat hippocampal neurons. One of these proteins, p-c-Raf, was upregulated in $\mathrm{Hu}-\mathrm{H} 1$-infected rats $(P<0.05)$. By contrast, p-MSK1 expression was decreased significantly in cells infected with both viruses compared with controls $(P<0.05)$. No significant changes in expression of p-90RSK, p-ERK1/2, p-MEK1/2, p-CREB, and BDNF were identified (Figure 6).

\section{Discussion}

$\mathrm{Hu}-\mathrm{H} 1$-infected rats inoculated at postnatal day 1 showed significantly decreased locomotor activity and exploration behavior by the OFT and the EPM test without motor dysfunction, which suggested that $\mathrm{Hu}-\mathrm{H} 1$ caused anxiety-like symptoms, while Strain V-infected rats displayed another abnormal behavior. Moreover, in characterizing members of the ERK/CREB/BDNF pathway in the hippocampi of rats infected with both BDV strains, we found that p-ERK1/2 was upregulated (Strain V- and Hu-H1-infected rats vs control rats) and other signaling molecules either increased (p-90RSK and p-MSK1 in Hu-H1-infected rats vs control rats, $P<0.05$; p-c-Raf in Strain V-infected rats vs control, $P<0.05$ ) or showed an increasing trend (p-MEK1/2, $\mathrm{p}$-CREB, CREB, and BDNF). Unlike these changes in vivo, expression of p-MSK1 $(P<0.05)$ was attenuated in primary hippocampal neurons infected with the two BDV strains. Together, these findings presented preliminary data that Hu-H1-infected and Strain V-infected rats displayed an anxiety-like phenotype or other abnormal behavior, which provided the basis for future study.

In a previous study, using a green fluorescent proteinexpressing BDV vector, BDV was found to disseminate in the mouse nervous system from the central to peripheral nervous system over time. ${ }^{35}$ Neonatal BDV-inoculated rats showed persistent infection without exhibiting clinical symptoms. ${ }^{36,37}$ In our study, BDV-infected rats had the same performance. In behavioral tests, on the one hand, $\mathrm{Hu}-\mathrm{H} 1$-infected rats indicated an anxiety-like phenotype that included significantly diminished total distance traveled, lower number of rearing events, and lower number of entries into center zone in the OFT as well as decreased total distance covered in the EPM test without motor dysfunction 


\section{Control}

A

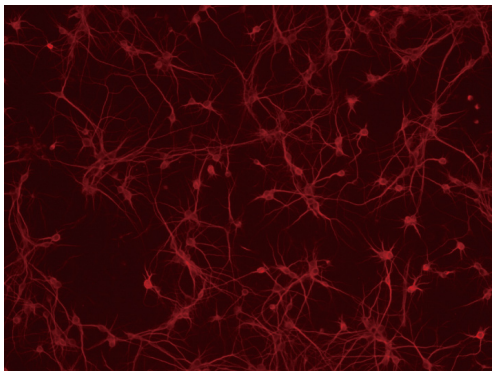

B

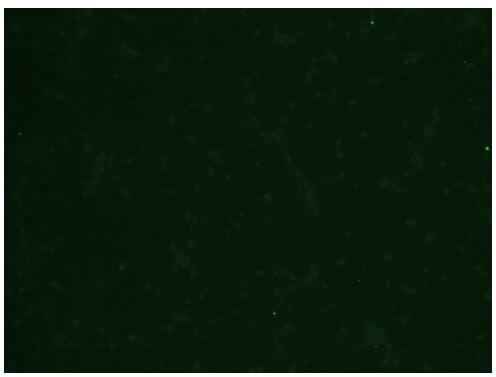

C

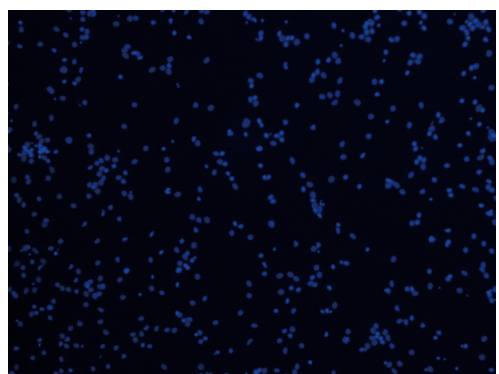

D

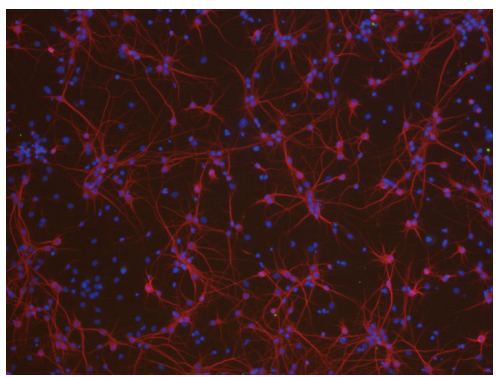

$\mathrm{Hu}-\mathrm{H} 1$
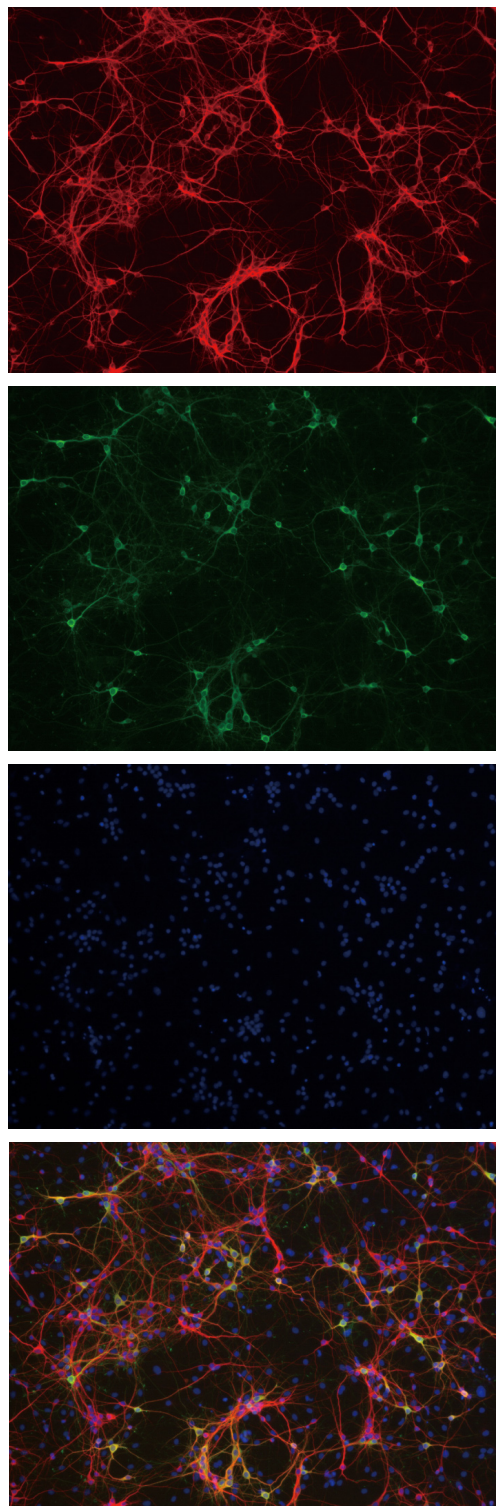

Strain V
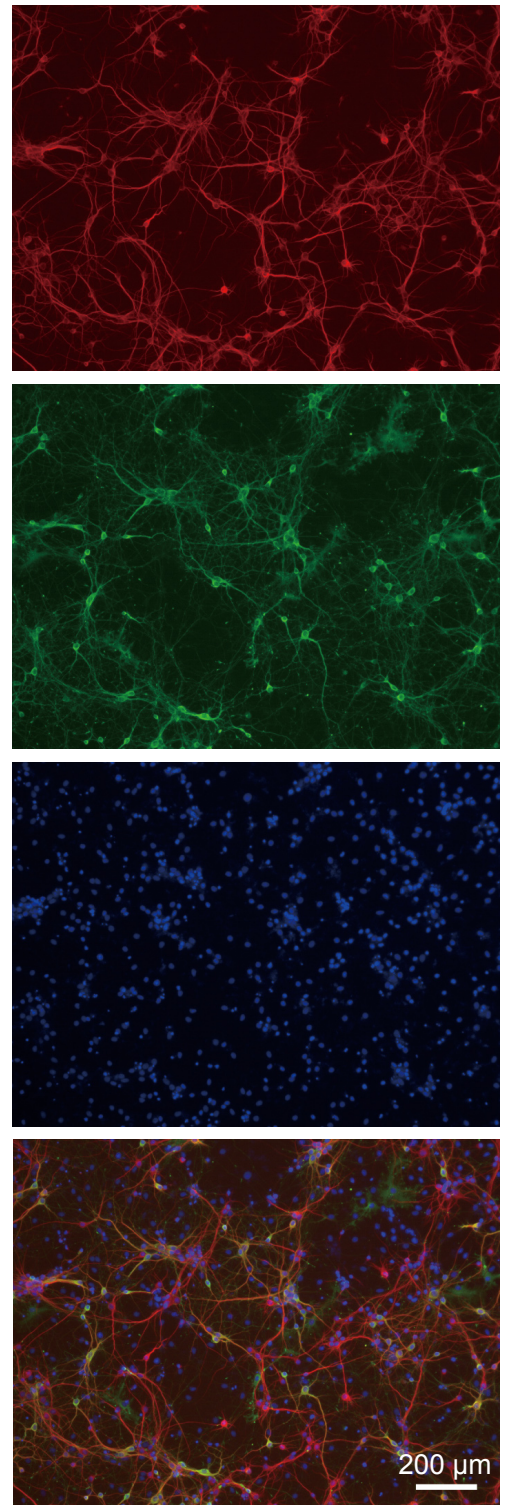

Figure 3 Immunofluorescence analysis of primary hippocampal neurons infected with BDV on day 9 postinfection.

Notes: (A) Purity of neurons stained with MAP2 followed by Cy3-labeled goat anti-rabbit lgG antibody (red). (B) BDV Hu-HI- or Strain V-infected cells combined with P24 antibody followed by FITC-labeled goat anti-mouse lgG antibody (green). (C) Nuclei of cells or DNA released from apoptotic cells were stained by DAPI (blue). (D) Merged images (magnification: I00x).

Abbreviation: BDV, Borna disease virus.

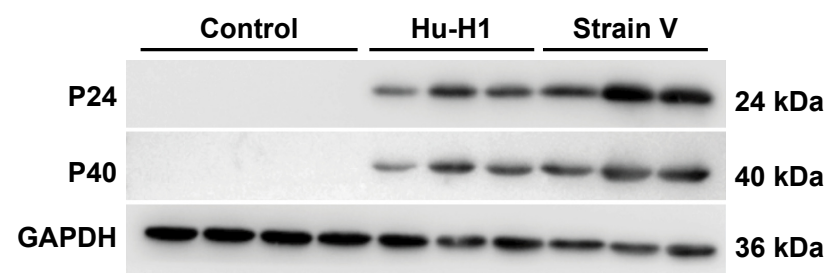

Figure 4 Identification of BDV infection in rats. BDV-specific antibodies (P24 and P40) identified the stable expression of virus in the hippocampus of rats 8 weeks postinfection compared with the control group.

Abbreviation: BDV, Borna disease virus. compared with the control group. On the other hand, Strain V-infected rats displayed abnormal behavior including lower numbers of rearing events in the OFT, decreased total distance covered, increased times, and entries into open arms in the EPM test as well as decreased immobile time in the FST compared with control rats. Similarly, previous studies demonstrated that CRP3 (BDV He-80, also horsederived)-infected neonatal Lewis rats persistently exhibited autistic-like behavior ${ }^{33,34,38}$ accompanied with impaired 

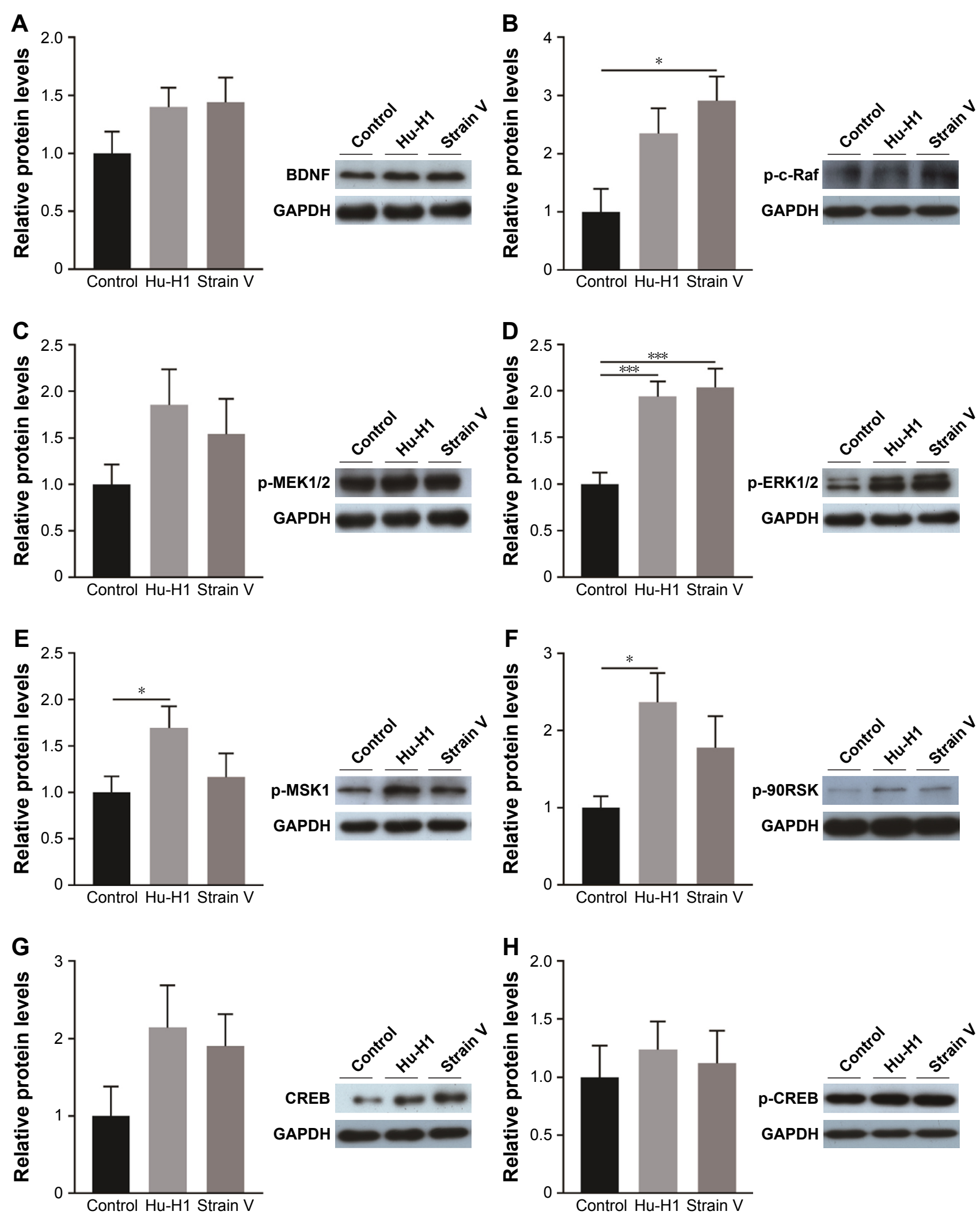

Figure 5 Western blotting of rat hippocampi.

Notes: Eight selected antibodies were included: $p-c-R a f, p-90 R S K, p-M S K, p-E R K I / 2, p-M E K I / 2, p-C R E B, C R E B$, and BDNF. Expression levels of (A) BDNF ( $n=7$ ), (C) p-MEKI/2 $(n=6),(\mathbf{G})$ CREB $(n=4)$, and $(\mathbf{H})$ p-CREB $(n=7)$ were not significantly affected by BDV infection. (B) Expression level of $p-c-R a f$ was upregulated in Strain $V$-infected rats compared with control rats $(P<0.05, n=4)$. (D) Expression level of $p$-ERKI/2 was significantly increased in $B D V$-infected rats relative to control rats $(P<0.00 \mathrm{I}$, $n=7)$. (E) Expression level of $p-M S K$ was significantly upregulated in Hu-HI-infected rats compared with control rats $(P<0.05, n=6)$. $(\mathbf{F})$ Expression level of $p$-90RSK was elevated in $\mathrm{Hu}-\mathrm{HI}$-infected rats compared with control rats $(P<0.05, \mathrm{n}=5)$. Bars indicate mean $\pm \mathrm{SEM}$. Statistical analysis was performed using ANOVA. $* P<0.05$ and $* * * P<0.001$.

Abbreviations: BDV, Borna disease virus; SEM, standard error of the mean. 
A
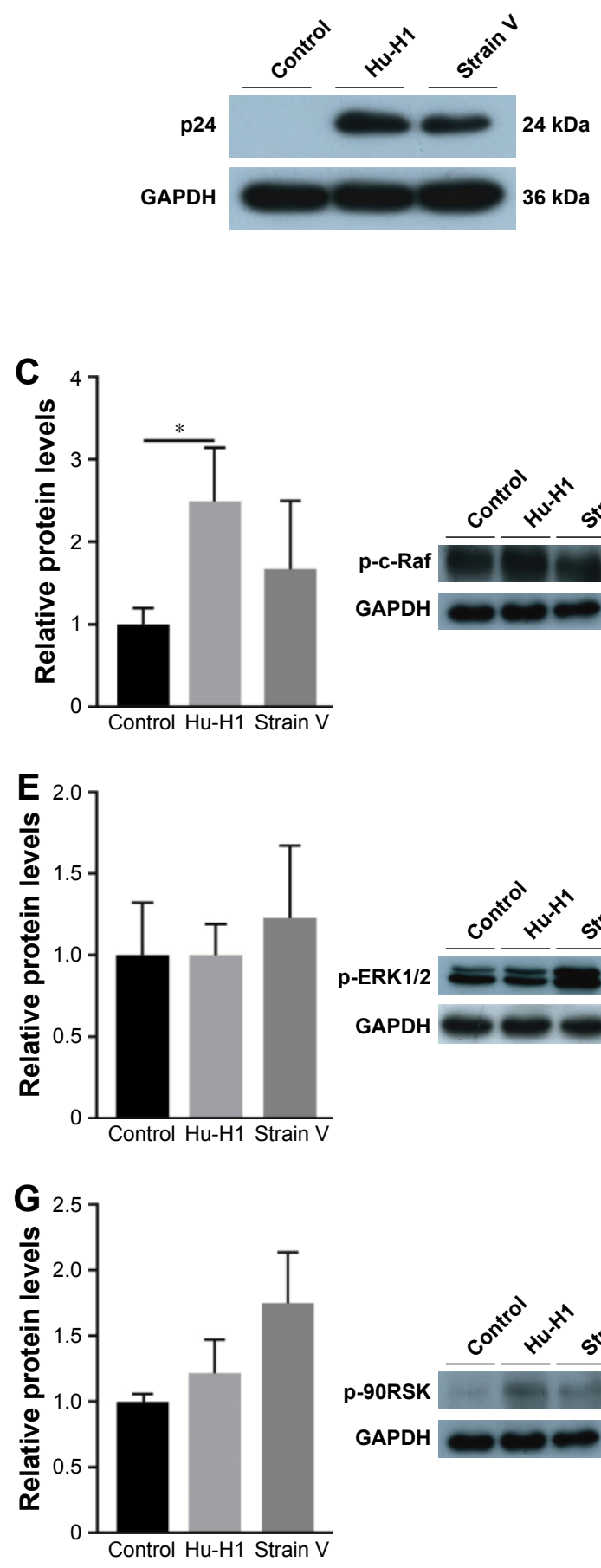
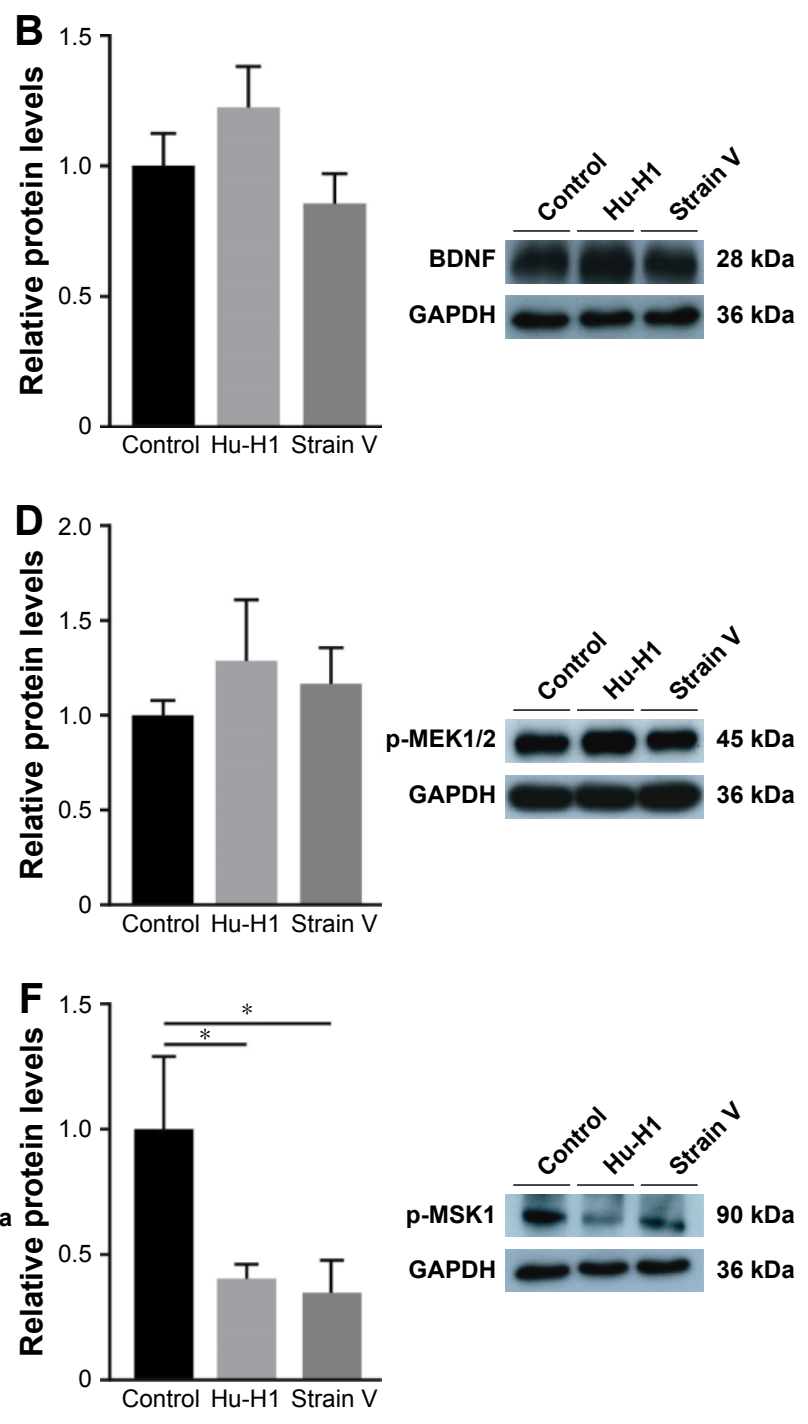

Figure 6 Western blotting of primary hippocampal neuron lysates cultured for 9 days.

Notes: (A): Identification of BDV infection in cells (A) No significant differences were found in the expression of (B) BDNF, (D) p-MEKI/2, (E) p-ERKI/2, (G) P-90RSK, and (H) P-CREB. (C) Expression level of $\mathrm{P}$-c-Raf was upregulated in Hu-HI-infected neurons compared with control neurons $(P<0.05)$. (F) Expression of $\mathrm{P}-\mathrm{MSK}$ was significantly downregulated in $\mathrm{Hu}-\mathrm{HI}$-infected and Strain $\mathrm{V}$-infected cells compared with mock-infected neurons $(P<0.05)$. The experiments were repeated thrice. Values were expressed as mean \pm SEM. $* P<0.05$.

Abbreviations: BDV, Borna disease virus; SEM, standard error of the mean.

cognitive functions, fear deficiency, hyperactivity, chronic anxiety, and decreased mobility. The most typical autism behavior phenotype were the social behavior deficiency and stereotyped behavior. ${ }^{39}$ However, our original intention was to observe depression or anxiety-like phenotype of rats based on existing experimental conditions, so we haven't performed the classical test to identify whether the Strain V-infected rats had autism-like phenotype. While Hu-H1 
inhibited proliferation and induced apoptosis in human oligodendrocytes cells, Strain V had an opposite effect. We took it into consideration that dissimilar disturbance in the energy metabolism and key amino acid metabolites between BDV Hu-H1 and Strain V had affected the apoptosis and proliferation of neurons or oligodendrocytes in the process of development of rats based on our previous study ${ }^{17,26,40}$ and the ERK pathway was correlated with this perturbation. Furthermore, even though $\mathrm{Hu}-\mathrm{H} 1$ and strain $\mathrm{V}$ shared a high degree of genetic homology, BDV strain, an old and evolutionary virus, ${ }^{41}$ exerted different biological effects in organisms. Overall, our experiment filled the gap in the behaviors of $\mathrm{Hu}-$ H1-infected rats. Although the same strain of BDV caused different behaviors, this deserves further study to understand the relationship between human mood disorders and BDV.

The ERK pathway plays a vital role in modulation of neuronal function, synaptic plasticity of neurons, cell survival, cell apoptosis, learning, and long-term potentiation. ${ }^{42}$ In this study, persistent infection of newborn rats resulted in significantly increased expression of p-ERK $1 / 2$ in the hippocampus (both BDV-infected groups vs control group). The ERK/ CREB/BDNF signaling cascade was activated in rats infected with BDV (Figure 7), which was consistent with previous reports showing that in CRL 1405 and OL cells, the cellular Raf/MEK/ERK was activated during infection with BDV $\mathrm{He} / 80 .{ }^{43}$ Nevertheless, in primary rat hippocampal neurons, expression of p-MSK1 was downregulated in cells infected with both BDV strains while p-c-Raf was upregulated in $\mathrm{Hu}-\mathrm{H} 1$-infeted neurons compared with uninfected neurons. These results were consistent with those of Liu et al, ${ }^{26}$ who found that BDV Hu-H1 activated the downstream ERK/RSK signalosome complex in OL cells. In our study, the various in vivo and in vitro results indicated that the ERK/CREB/ BDNF pathway was under complex regulatory control in the hippocampi of SD rats, and so further experiments are required to validate the effects of $\mathrm{BDV}$ on these signaling cascades.

$\mathrm{MEK} / \mathrm{ERK}$, as classical members of the MAPK pathway, is involved in virus replication and controls the expression of inflammatory cytokines. ${ }^{44}$ Similar activation of the Raf/ MEK/ERK pathway during replication and transcription of influenza virus was observed. ${ }^{45}$ However, the MEK-specific inhibitor U0126 could block virus spread to neighboring cells. ${ }^{43}$ Additionally, a previous study reported that the MEK inhibitor U0126 showed antiviral activity against H1N1 (pandemic swine influenza A virus) and highly pathogenic

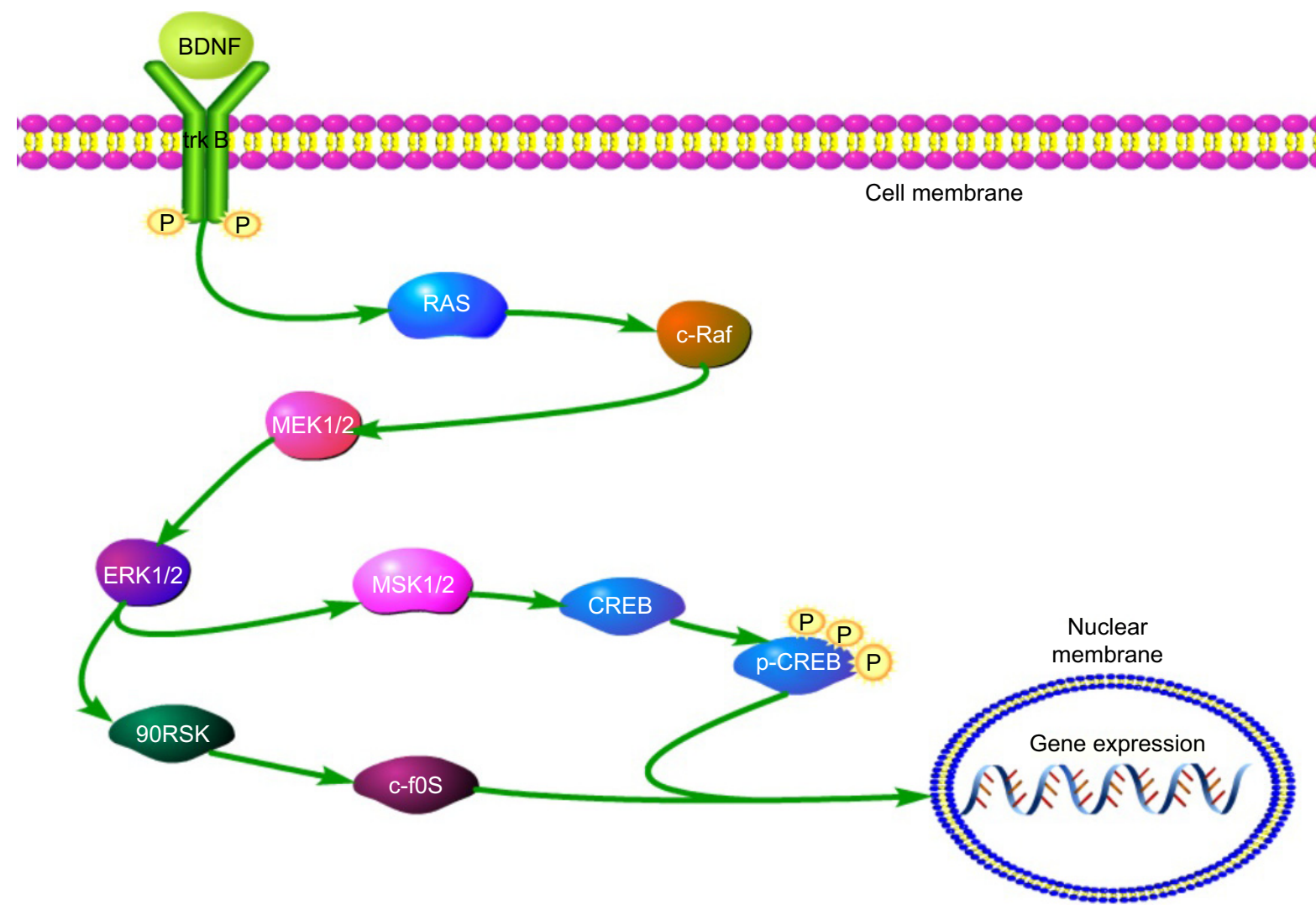

Figure 7 The ERK/CREB/BDNF cascade in this study. 
avian influenza viruses both in vitro and in vivo without obvious adverse effects. ${ }^{46}$ This sheds new light on potential investigations of antiviral drugs for inhibiting inter-cell BDV transport in the future. Accordingly, considering the evidence indicating that BDV may be associated with schizophrenia or depression, ${ }^{47-50}$ studies of BDV could provide a valuable model to investigate the neuropathogenesis of human mental disorders.

\section{Conclusion}

Taken together, our data provided preliminary evidences that BDV Hu-H1-infected and strain V-infected rats developed anxiety-like and an abnormal behavior, respectively, which was relevant to activation of the ERK/CREB/BDNF signaling pathway. Several limitations of our study should be mentioned. Firstly, a comparatively small sample size was used. Secondly, the mechanisms of BDV-associated behavioral modification were not deeply elucidated and we have not performed the classical social behavior test to detect whether the Strain V-infected rats had autism-like phenotype. Further studies examining modulation of the ERK/CREB/BDNF signaling cascade as well as amantadine or 5-HT receptor inhibitors on the behavior of BDV-infected rats may provide further insights into the relevance of the behavioral disturbances in these rats for human neuropsychiatric disorders.

\section{Acknowledgments}

This study was financially supported by the National Key Research and Development Program of China (Grant No 2017YFA0505700), the Young Project of National Natural Science Foundation of China (Grant No 81601207), the Scientific and Technological Research Program of Chongqing Municipal Education Commission (Grant No KJ1600223), and the Basic and Frontier Exploration Project of Chongqing (Grant No CSTC2016JCYJA0159 and Grant No CSTC2015JCYJA10081). We thank Dr Jian Lu for his assistance with graphics. We thank Liwen Bianji, Edanz Editing, China, for editing the English text of the draft of this manuscript.

\section{Disclosure}

The authors report no conflicts of interest in this work.

\section{References}

1. Kinnunen PM, Palva A, Vaheri A, Vapalahti O. Epidemiology and host spectrum of Borna disease virus infections. J Gen Virol. 2013;94(Pt 2): 247-262.

2. Staeheli P, Sauder C, Hausmann J, Ehrensperger F, Schwemmle M. Epidemiology of Borna disease virus. J Gen Virol. 2000;81(Pt 9):2123-2135.
3. Bode L, Dietrich DE, Stoyloff R, Emrich HM, Ludwig H. Amantadine and human Borna disease virus in vitro and in vivo in an infected patient with bipolar depression. Lancet. 1997;349(9046):178-179.

4. Deuschle M, Bode L, Heuser I, Schmider J, Ludwig H. Borna disease virus proteins in cerebrospinal fluid of patients with recurrent depression and multiple sclerosis. Lancet. 1998;352(9143):1828-1829.

5. Hoffmann B, Tappe D, Höper D, et al. A Variegated Squirrel Bornavirus Associated with Fatal Human Encephalitis. $N$ Engl J Med. 2015; 373(2):154-162.

6. Honda $\mathrm{T}$, Sofuku K, Matsunaga $\mathrm{H}$, et al. Detection of Antibodies against Borna Disease Virus Proteins in an Autistic Child and Her Mother. Jpn J Infect Dis. 2017;70(5):599.

7. Salvatore M, Morzunov S, Schwemmle M, Lipkin WI. Borna disease virus in brains of North American and European people with schizophrenia and bipolar disorder. Bornavirus Study Group. Lancet. 1997; 349(9068):1813-1814.

8. Soltani H, Mohammadzadeh S, Makvandi M, Pakseresht S, SamarbafZadeh A. Detection of Borna Disease Virus (BDV) in Patients with First Episode of Schizophrenia. Iran J Psychiatry. 2016;11(4):257-261.

9. Zaliunaite V, Steibliene V, Bode L, Podlipskyte A, Bunevicius R, Ludwig H. Primary psychosis and Borna disease virus infection in Lithuania: a case control study. BMC Psychiatry. 2016;16(1):369.

10. Billaud JN, Ly C, Phillips TR, de La Torre JC. Borna disease virus persistence causes inhibition of glutamate uptake by feline primary cortical astrocytes. $J$ Virol. 2000;74(22):10438-10446.

11. Ovanesov MV, Vogel MW, Moran TH, Pletnikov MV. Neonatal Borna disease virus infection in rats is associated with increased extracellular levels of glutamate and neurodegeneration in the striatum. J Neurovirol. 2007;13(3):185-194.

12. Solbrig MV, Koob GF, Joyce JN, Lipkin WI. A neural substrate of hyperactivity in borna disease: changes in brain dopamine receptors. Virology. 1996;222(2):332-338.

13. Stitz L, Dietzschold B, Carbone KM. Immunopathogenesis of Borna disease. Curr Top Microbiol Immunol. 1995;190:75-92.

14. Tizard I, Ball J, Stoica G, Payne S. The pathogenesis of bornaviral diseases in mammals. Anim Health Res Rev. 2016;17(2):92-109.

15. Zhang L, Lei Y, Liu X, et al. Glutamate and lipid metabolic perturbation in the hippocampi of asymptomatic borna disease virus-infected horses. PLoS One. 2014;9(6):e99752.

16. Bode L, Dürrwald R, Rantam FA, Ferszt R, Ludwig H. First isolates of infectious human Borna disease virus from patients with mood disorders. Mol Psychiatry. 1996;1(3):200-212.

17. Li D, Lei Y, Deng J, et al. Human but Not Laboratory Borna Disease Virus Inhibits Proliferation and Induces Apoptosis in Human Oligodendrocytes In Vitro. PLoS One. 2013;8(6):e66623.

18. Ludwig H, Bode L. Borna disease virus: new aspects on infection, disease, diagnosis and epidemiology. Rev Sci Tech. 2000;19(1):259-288.

19. Planz O, Pleschka S, Wolff T. Borna disease virus: a unique pathogen and its interaction with intracellular signalling pathways. Cell Microbiol. 2009;11(6):872-879.

20. Pleschka S. RNA viruses and the mitogenic Raf/MEK/ERK signal transduction cascade. Biol Chem. 2008;389(10):1273-1282.

21. Kyosseva SV. Differential expression of mitogen-activated protein kinases and immediate early genes fos and jun in thalamus in schizophrenia. Prog Neuropsychopharmacol Biol Psychiatry. 2004;28(6): 997-1006.

22. Duman CH, Schlesinger L, Kodama M, Russell DS, Duman RS. A role for MAP kinase signaling in behavioral models of depression and antidepressant treatment. Biol Psychiatry. 2007;61(5):661-670.

23. Yufune S, Satoh Y, Takamatsu I, et al. Transient Blockade of ERK Phosphorylation in the Critical Period Causes Autistic Phenotypes as an Adult in Mice. Sci Rep. 2015;5:10252.

24. Subramanian M, Timmerman CK, Schwartz JL, Pham DL, Meffert MK Characterizing autism spectrum disorders by key biochemical pathways. Front Neurosci. 2015;9:313.

25. Manji HK, Drevets WC, Charney DS. The cellular neurobiology of depression. Nat Med. 2001;7(5):541-547. 
26. Liu X, Yang Y, Zhao M, et al. Proteomics reveal energy metabolism and mitogen-activated protein kinase signal transduction perturbation in human Borna disease virus $\mathrm{Hu}-\mathrm{H} 1$-infected oligodendroglial cells. Neuroscience. 2014;268:284-296.

27. Hans A, Syan S, Crosio C, Sassone-Corsi P, Brahic M, Gonzalez-Dunia D. Borna disease virus persistent infection activates mitogen-activated protein kinase and blocks neuronal differentiation of PC12 cells. J Biol Chem. 2001;276(10):7258-7265.

28. Zhao M, Sun L, Chen S, et al. Borna disease virus infection impacts microRNAs associated with nervous system development, cell differentiation, proliferation and apoptosis in the hippocampi of neonatal rats. Mol Med Rep. 2015;12(3):3697-3703.

29. Cheng K, Li J, Yang D, et al. 2D-gel based proteomics unravels neurogenesis and energetic metabolism dysfunction of the olfactory bulb in CUMS rat model. Behav Brain Res. 2016;313:302-309.

30. Liu YY, Zhou XY, Yang LN, et al. Social defeat stress causes depressionlike behavior with metabolite changes in the prefrontal cortex of rats. PLoS One. 2017;12(4):e0176725.

31. Zhang L, Liu S, Zhang L, et al. Real-time qPCR identifies suitable reference genes for Borna disease virus-infected rat cortical neurons. Int J Mol Sci. 2014;15(12):21825-21839.

32. Mao Q, Zhang L, Guo Y, et al. Identification of suitable reference genes for BDV-infected primary rat hippocampal neurons. Mol Med Rep. 2016;14(6):5587-5594.

33. Lancaster K, Dietz DM, Moran TH, Pletnikov MV. Abnormal social behaviors in young and adult rats neonatally infected with Borna disease virus. Behav Brain Res. 2007;176(1):141-148.

34. Pletnikov MV, Rubin SA, Vasudevan K, Moran TH, Carbone KM. Developmental brain injury associated with abnormal play behavior in neonatally Borna disease virus-infected Lewis rats: a model of autism. Behav Brain Res. 1999;100(1-2):43-50.

35. Ackermann A, Guelzow T, Staeheli P, Schneider U, Heimrich B. Visualizing viral dissemination in the mouse nervous system, using a green fluorescent protein-expressing Borna disease virus vector. $J$ Virol. 2010;84(10):5438-5442.

36. Hirano N, Kao M, Ludwig H, Persistent LH. Persistent, tolerant or subacute infection in Borna disease virus-infected rats. J Gen Virol. 1983; 64 (Pt 7):1521-1530.

37. Hornig M, Weissenböck H, Horscroft N, Lipkin WI. An infection-based model of neurodevelopmental damage. Proc Natl Acad Sci US A. 1999; 96(21):12102-12107.
38. Hornig M, Solbrig M, Horscroft N, Weissenböck H, Lipkin WI. Borna disease virus infection of adult and neonatal rats: models for neuropsychiatric disease. Curr Top Microbiol Immunol. 2001;253:157-177.

39. Ferhat AT, Halbedl S, Schmeisser MJ, Kas MJ, Bourgeron T, Ey E. Behavioural Phenotypes and Neural Circuit Dysfunctions in Mouse Models of Autism Spectrum Disorder. Adv Anat Embryol Cell Biol. 2017;224:85-101

40. Liu S, Bode L, Zhang L, et al. GC-MS-Based Metabonomic Profiling Displayed Differing Effects of Borna Disease Virus Natural Strain $\mathrm{Hu}-\mathrm{H} 1$ and Laboratory Strain V Infection in Rat Cortical Neurons. Int J Mol Sci. 2015;16(8):19347-19368.

41. Horie M, Honda T, Suzuki Y, et al. Endogenous non-retroviral RNA virus elements in mammalian genomes. Nature. 2010;463(7277):84-87.

42. Kyosseva SV. The role of the extracellular signal-regulated kinase pathway in cerebellar abnormalities in schizophrenia. Cerebellum. 2004; 3(2):94-99.

43. Planz O, Pleschka S, Ludwig S. MEK-specific inhibitor U0126 blocks spread of Borna disease virus in cultured cells. J Virol. 2001;75(10): $4871-4877$.

44. Bruder JT, Kovesdi I. Adenovirus infection stimulates the Raf/MAPK signaling pathway and induces interleukin-8 expression. J Virol. 1997; 71(1):398-404

45. Marjuki H, Alam MI, Ehrhardt C, et al. Membrane accumulation of influenza A virus hemagglutinin triggers nuclear export of the viral genome via protein kinase Calpha-mediated activation of ERK signaling. J Biol Chem. 2006;281(24):16707-16715.

46. Droebner K, Pleschka S, Ludwig S, Planz O. Antiviral activity of the MEK-inhibitor U0126 against pandemic H1N1v and highly pathogenic avian influenza virus in vitro and in vivo. Antiviral Res. 2011;92(2): 195-203.

47. Heinrich A, Adamaszek M. Anti-Borna disease virus antibody responses in psychiatric patients: long-term follow up. Psychiatry Clin Neurosci. 2010;64(3):255-261.

48. Nunes SO, Itano EN, Amarante MK, et al. RNA from Borna disease virus in patients with schizophrenia, schizoaffective patients, and in their biological relatives. J Clin Lab Anal. 2008;22(4):314-320.

49. Wang X, Zhang L, Lei Y, et al. Meta-analysis of infectious agents and depression. Sci Rep. 2014;4:4530.

50. Zhang L, Xu MM, Zeng L, et al. Evidence for Borna disease virus infection in neuropsychiatric patients in three western China provinces. Eur J Clin Microbiol Infect Dis. 2014;33(4):621-627.
Neuropsychiatric Disease and Treatment

\section{Publish your work in this journal}

Neuropsychiatric Disease and Treatment is an international, peerreviewed journal of clinical therapeutics and pharmacology focusing on concise rapid reporting of clinical or pre-clinical studies on a range of neuropsychiatric and neurological disorders. This journal is indexed on PubMed Central, the 'PsycINFO' database and CAS,
Dovepress

and is the official journal of The International Neuropsychiatric Association (INA). The manuscript management system is completely online and includes a very quick and fair peer-review system, which is all easy to use. Visit http://www.dovepress.com/testimonials.php to read real quotes from published authors. 